\title{
Changes of Locomotion Speed Affect Distance Estimations in Virtual Reality
}

\author{
Julian Keil ${ }^{a}$ **, Annika Korte ${ }^{\text {a }}$, Dennis Edler ${ }^{\text {a }}$, Denise O`Meara ${ }^{\text {a }}$, Frank Dickmann ${ }^{\text {a }}$ \\ ${ }^{a}$ Ruhr UniversityBochum, Germany,julian.keil@rub.de, annika.korte@rub.de,dennis.edler@rub.de,denise.omeara@rub.de, \\ frank.dickmann@rub.de \\ * Corresponding author
}

\begin{abstract}
Modern Virtual Reality (VR) applications often use artificial locomotion to allow users to travel distances within VR space that exceed the available space used to transfer real-world and real-time motion into the virtual environment. The locomotion speed is usually not fixed and can be selected dynamically by the user. Due to motion adaptation effects, variations of locomotion speed could affect how distances in VR are perceived. In the context of cartographic VR applications aimed to experience and communicate spatial information, such effects on distance perception could be problematic, because they might lead to distortions in cognitive representations of space acquired via interaction with VR environments. By conducting a VR-based distance estimation study, we demonstrate how changes of artificial locomotion speed affect distance estimations in VR. Increasing locomotion speeds after letting users adapt to a lower locomotion speed led to lower distance estimations and decreasing locomotion speeds led to higher distance estimations. These findings should sensitize VR developers to consider the choice of applied locomotion techniques when a developed VR application is supposed to communicate distance information or to support the acquisition of a cognitive representation of geographic space.
\end{abstract}

Keywords: virtual reality, distance estimations, artificial locomotion, locomotion speed, motion adaptation

\section{Introduction}

The use of modern Virtual Reality (VR) technology allows cartographers to easily represent geographic space in $3 \mathrm{D}$ maps in a 1:1 scale (Hruby et al., 2020; Keil, Edler, Schmitt, \& Dickmann, 2021). Such VR-based spatial representations provide the opportunity to explore a virtual simulation of geographic space and to build a cognitive representation of the explored space without direct realworld perception. However, despite technological advances, VR-based interaction with 3D space is still not similar to interaction with real-world space (Boletsis \& Cedergren, 2019). Due to technical limitations of the available spatial tracking systems and their constraints on the tracking area, locomotion in VR applications for traveling farther than a few meters is usually based on controller input. Two of the most prominent examples are teleportation and (continuous) artificial locomotion. Teleportation requires the user to aim at a target location with a controller while a button is pressed down. When the button is released, the user is instantly relocated to the selected target location. Artificial locomotion allows users to select a travel direction using touchpad or analog stick (also called thumbstick) input. Based on the controller input, the user is translated continuously forward into the selected direction within VR space. Which locomotion technique is applied to a VR application can affect how VR space is perceived (Boletsis \& Cedergren, 2019). For example, as demonstrated by Keil, Edler, O'Meara, et al. (2021), distances traveled within VR space are perceived differently if artificial locomotion is applied compared to teleportation. Providing a distance estimation training improved the accuracy of distance estimations, but the improvements varied based on the applied locomotion technique. These findings demonstrate that the selection of an appropriate locomotion technique is essential for the conveyance of spatial information via VR, because cognitive representations of geographic space acquired via interaction with a VR environment can be distorted if distances between object pairs are underestimated or overestimated.

When developers of VR applications implement artificial locomotion, they can define a locomotion speed limit. Although this restricts the ability to travel quickly through VR space, specifying an upper speed limit is highly important for the comfort of the user. The discrepancy between visually perceived locomotion and vestibular or proprioceptive cues can cause VR sickness (Langbehn et al., 2018). This discrepancy increases with higher 
locomotion speeds leading to higher levels of discomfort and nausea (So et al., 2001). In addition to an upper speed limit, developers can determine whether the locomotion speed is fixed or whether the user can dynamically select locomotion speeds between zero and the predefined upper speed limit.

The potential ability of users to select an artificial locomotion speed raises the question how speed variations affect spatial perception and distance estimations within VR space. Hietanen et al. (2008) demonstrated that, due to motion adaptation, perceived speeds purely based on visual (optic flow) input from a computer screen are affected by the speed of previously received visual information. Speeds of visual stimuli were found to be perceived as faster if speeds of previously presented visual stimuli were slower. However, similar to previous studies on screen-based motion adaptation effects (Hoffmann et al., 1999; Schrater \& Simoncelli, 1998), the study of Hietanen et al. (2008) provided optic flow information from abstract stimuli instead of self-motion-based visual input. Gray and Regan (2000) found evidence for effects of self-motion adaptation on speed perception, but the study was single screen-based, the locomotion was not self-controlled and distance estimations were not assessed. If the effects of locomotion adaptation can be generalized to the perception of self-controlled self-motion in stereoscopically perceived VR environments, it can be assumed that the ability of VR users to adjust artificial locomotion speeds dynamically affects the accuracy of speed perception and, consequentially, the accuracy of distance estimations in VR environments.

The study reported in this paper investigates this assumption by applying a distance estimation task in a VR environment with varying artificial locomotion speeds. Based on the findings of Hietanen et al. (2008), we assume that increasing the artificial locomotion speed after applying a slower artificial locomotion speed should disproportionately increase estimations of subjective locomotion speed and, consequentially, increase estimations of the traveled distance. Furthermore, we would expect that a distance estimation training as used by Keil, Edler, O'Meara, et al. (2021) is less suitable to reduce distance estimation errors if different locomotion speeds are applied, because they can deviate from the speed applied during the distance estimation training. The general purpose of the study is to broaden our understanding of how locomotion techniques affect spatial perception within VR applications. Especially for applications aimed at conveying spatial relations and forming accurate cognitive representations of space, identifying and correcting design choices that cause systematic distortions of cognitive representations of space is essential.

\section{Methods}

The study was controlled and approved by the ethics committee of the Faculty of Geosciences at the Ruhr University Bochum.

\subsection{Participants}

The study sample consisted of 96 students (37 female, 59 male) from Ruhr University Bochum. The age in the sample ranged between 19 and 41 years $(\mathrm{M}=25.5, \mathrm{SD}=$ 3.8). The required sample size was determined using G*Power version 3.1.9.7 (three groups, Wilcoxon signedrank test with matched pairs, effect size $=0.05, \alpha$ error probability $=0.05$, power $=0.85)$. Participation was rewarded with a payment of five Euro.

\subsection{Apparatus}

A virtual 3D corridor (see Figure 1) was created using the Unity game engine (version 2019.1.0f2). On both sides, the corridor was confined by $2.25 \mathrm{~m}$ high walls covered with a solid green texture. The corridor between the walls was $4 \mathrm{~m}$ wide and covered with a $1 \mathrm{~m}$ wide sand texture in the center of the corridor and a shrub texture on the other areas of the floor. Via code, a red dot marking a target location could be placed on top of the white sand texture. Tracking of a VR HMD and controller was implemented using the SteamVR asset from Valve Corporation. The used VR hardware consisted of two Valve Base Stations 2.0 for spatial tracking, an HTC Vive Pro HMD and an HTC Vive Pro controller. A continuous forward locomotion (either 3,5 or $7 \mathrm{~km} / \mathrm{h}$ ) was assigned to the upper $40 \%$ touchpad area of the controller (see Figure 2).

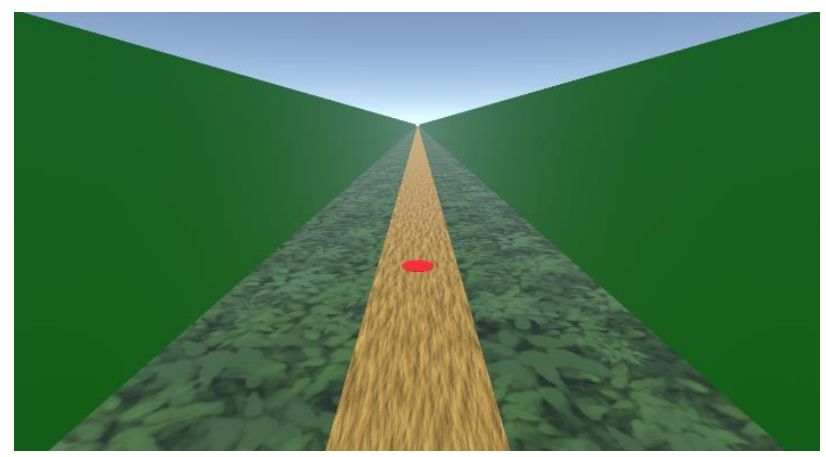

Figure 1. Virtual corridor. Participants were asked to travel towards the red target dot using artificial locomotion and to estimate the traveled distance. 


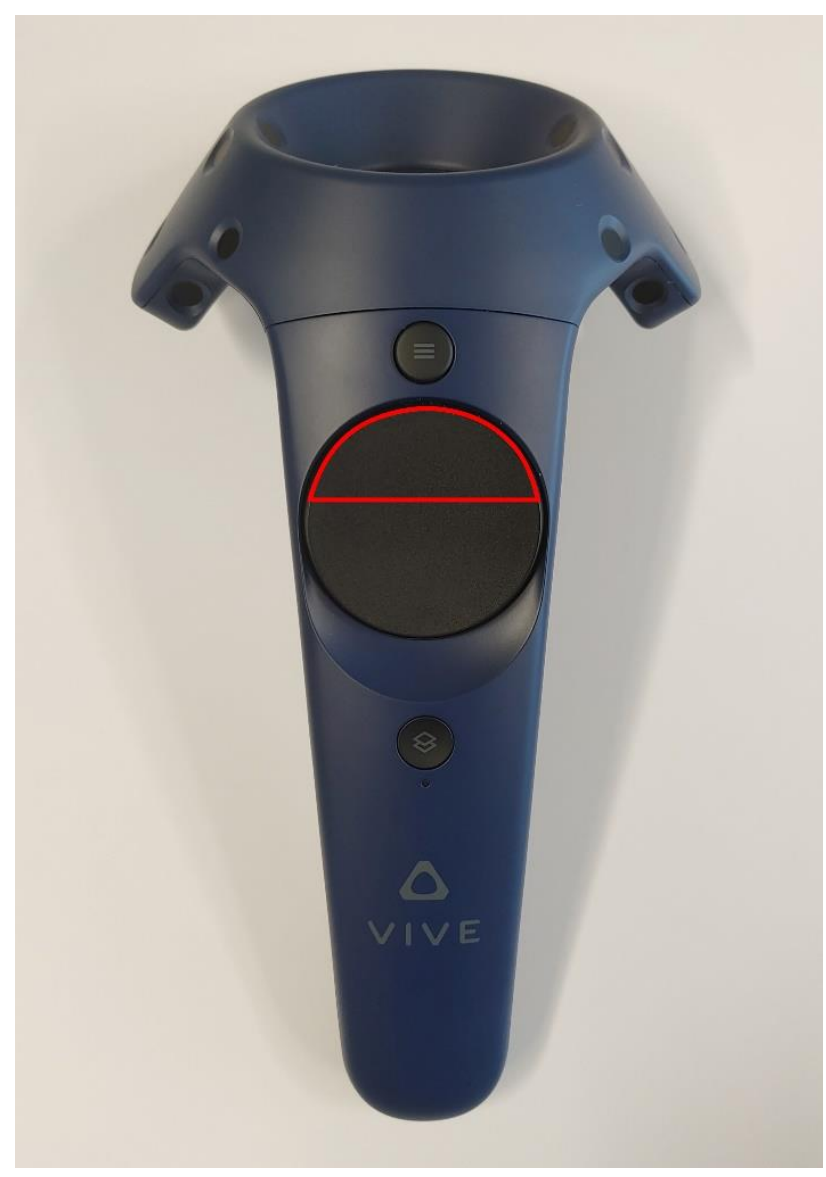

Figure 2. Controller input. By touching the upper $40 \%$ of the touchpad marked by the red semicircle, participants were able to deploy an artificial forward locomotion.

\subsection{Procedure}

The experiment consisted of three distance estimation trials with three between-subject conditions used to vary the artificial locomotion speed across the trials (condition 1: $5 / 5 / 5 \mathrm{~km} / \mathrm{h}$, condition $2: 5 / 7 / 3 \mathrm{~km} / \mathrm{h}$, condition $3: 5 / 3 / 7$ $\mathrm{km} / \mathrm{h}$ ). A target represented by a red dot (see Figure 1) was visually highlighted at a random location between 30 and $60 \mathrm{~m}$ from the start location. Participants were required to travel to this dot using artificial locomotion and then asked to give a verbal estimate of the traveled distance. Between trial one and two, as well as trial two and three, a distance estimation training with an artificial locomotion speed of $5 \mathrm{~km} / \mathrm{h}$ was provided. In these trainings, participants traveled to a target located $10 \mathrm{~m}$ from the start location and were informed about the traveled distance.

\subsection{Measures}

To investigate potential underestimations and overestimations of traveled distances, directional distance estimation errors were calculated based on the verbal estimates and the correct distances. Negative values represent underestimations and positive values represent overestimations. Furthermore, to assess the general spread of underestimations and overestimations, absolute distance estimation errors were calculated. Both error values were assessed separately for each participant and trial.

\subsection{Statistics}

Due to the non-normal distribution of the data, Wilcoxon signed-rank tests were used to compare directional and absolute distance estimation errors between the first and second distance estimation trial, as well as the second and third distance estimation trial. Tests were carried out separately per between-subject condition.

\section{Results}

In the between-subject condition with constant locomotion speeds $(5 / 5 / 5 \mathrm{~km} / \mathrm{h})$, no significant differences of directional distance estimations were found between the first and second trial $\left(\mathrm{M}_{\text {Trial1 }}=-6.44 \mathrm{~m}, \mathrm{SD}_{\text {Trial1 }}=28.15 \mathrm{~m}\right.$, $\mathrm{M}_{\text {Trial2 }}=-0.5 \mathrm{~m}, \mathrm{SD}_{\text {Trial2 }}=22.79 \mathrm{~m}, \mathrm{Z}=1.05, \mathrm{p}=.29$ ) or the second and third trial $\left(\mathrm{M}_{\text {Trial2 }}=-0.5 \mathrm{~m}, \mathrm{SD}_{\text {Trial2 }}=22.79\right.$ $\left.\mathrm{m}, \mathrm{M}_{\text {Trial3 }}=-0.88 \mathrm{~m}, \mathrm{SD}_{\text {Trial3 }}=12.52 \mathrm{~m}, \mathrm{Z}=-1.17, \mathrm{p}=.24\right)$. However, absolute errors in trial two were lower than in trial one $\left(\mathrm{M}_{\text {Trial } 1}=19.75 \mathrm{~m}, \mathrm{SD}_{\text {Trial1 }}=20.8 \mathrm{~m}, \mathrm{M}_{\text {Trial2 }}=\right.$ $12.75 \mathrm{~m}, \mathrm{SD}_{\text {Trial } 2}=18.75 \mathrm{~m}, \mathrm{Z}=-2.46, \mathrm{p}=.01$, see Figure $3)$. Absolute errors in trial three were even lower than in trial two, but the difference barely missed statistical significance $\left(\mathrm{M}_{\text {Trial2 }}=12.75 \mathrm{~m}, \mathrm{SD}_{\text {Trial2 }}=18.75 \mathrm{~m}, \mathrm{M}_{\text {Trial } 3}\right.$ $\left.=7.88 \mathrm{~m}, \mathrm{SD}_{\text {Trial3 }}=9.67 \mathrm{~m}, \mathrm{Z}=-1.84, \mathrm{p}=.06\right)$.

In the second condition $(5 / 7 / 3 \mathrm{~km} / \mathrm{h})$, directional errors did not significantly change when the locomotion speed was increased to seven $\mathrm{km} / \mathrm{h}$ in the second trial $\left(\mathrm{M}_{\text {Trial1 }}=-5.44\right.$ $\mathrm{m}, \mathrm{SD}_{\text {Trial1 }}=25.58 \mathrm{~m}, \mathrm{M}_{\text {Trial2 }}=-7.31 \mathrm{~m}, \mathrm{SD}_{\text {Trial2 }}=16.42 \mathrm{~m}$, $\mathrm{Z}=-0.41, \mathrm{p}=.68$ ), but a significant shift from underestimations to overestimations was observed when the locomotion speed was decreased to three $\mathrm{km} / \mathrm{h}$ in the third trial $\left(\mathrm{M}_{\text {Trial2 }}=-7.31 \mathrm{~m}, \mathrm{SD}_{\text {Trial2 }}=16.42 \mathrm{~m}, \mathrm{M}_{\text {Trial } 3}=\right.$ $\left.4.66 \mathrm{~m}, \mathrm{SD}_{\text {Trial3 }}=34.41 \mathrm{~m}, \mathrm{Z}=2.11, \mathrm{p}=.04\right)$. Absolute errors were significantly lower in trial two than in trial one $\left(\mathrm{M}_{\text {Trial1 }}=21.38 \mathrm{~m}, \mathrm{SD}_{\text {Trial1 }}=14.6 \mathrm{~m}, \mathrm{M}_{\text {Trial2 }}=13.56 \mathrm{~m}\right.$, $\left.\mathrm{SD}_{\text {Trial2 }}=11.62 \mathrm{~m}, \mathrm{Z}=-2.39, \mathrm{p}=.02\right)$. In trial three, absolute errors were much higher than in trial two, but the difference was not statistically significant. This is potentially due to the high response variance in trial three $\left(\mathrm{M}_{\text {Trial2 }}=13.56 \mathrm{~m}, \mathrm{SD}_{\text {Trial2 }}=11.62 \mathrm{~m}, \mathrm{M}_{\text {Trial3 }}=20.59 \mathrm{~m}\right.$, $\mathrm{SD}_{\text {Trial } 3}=27.73 \mathrm{~m}, \mathrm{Z}=1.03, \mathrm{p}=.3$ ).

In the third condition $(5 / 3 / 7 \mathrm{~km} / \mathrm{h})$, directional distance estimation errors shifted from underestimations to overestimations when the locomotion speed was decreased from five to three $\mathrm{km} / \mathrm{h}$ in trial two $\left(\mathrm{M}_{\text {Trial1 }}=-9 \mathrm{~m}, \mathrm{SD}_{\text {Trial } 1}\right.$ $=23.22 \mathrm{~m}, \mathrm{M}_{\text {Trial2 }}=2.72 \mathrm{~m}, \mathrm{SD}_{\text {Trial2 }}=18.26 \mathrm{~m}, \mathrm{Z}=2.76$, $\mathrm{p}<.01)$. When the locomotion speed was increased to seven $\mathrm{km} / \mathrm{h}$ in trial three, directional distance estimations shifted back to underestimations $\left(\mathrm{M}_{\text {Trial2 }}=2.72 \mathrm{~m}, \mathrm{SD}_{\text {Trial2 }}\right.$ $=18.26 \mathrm{~m}, \mathrm{M}_{\text {Trial3 }}=-8.66 \mathrm{~m}, \mathrm{SD}_{\text {Trial } 3}=11.9 \mathrm{~m}, \mathrm{Z}=-2.96$, $\mathrm{p}<.01)$. Absolute errors decreased in trial two $\left(\mathrm{M}_{\text {Trial1 }}=\right.$ 
$20 \mathrm{~m}, \mathrm{SD}_{\text {Trial1 }}=14.48 \mathrm{~m}, \mathrm{M}_{\text {Trial2 }}=14.78 \mathrm{~m}, \mathrm{SD}_{\text {Trial2 }}=10.75$ $\mathrm{m}, \mathrm{Z}=-1.27, \mathrm{p}=.2)$ and in trial three $\left(\mathrm{M}_{\text {Trial2 }}=14.78 \mathrm{~m}\right.$, $\mathrm{SD}_{\text {Trial2 }}=10.75 \mathrm{~m}, \mathrm{M}_{\text {Trial3 }}=12.47 \mathrm{~m}, \mathrm{SD}_{\text {Trial3 }}=7.65 \mathrm{~m}, \mathrm{Z}$

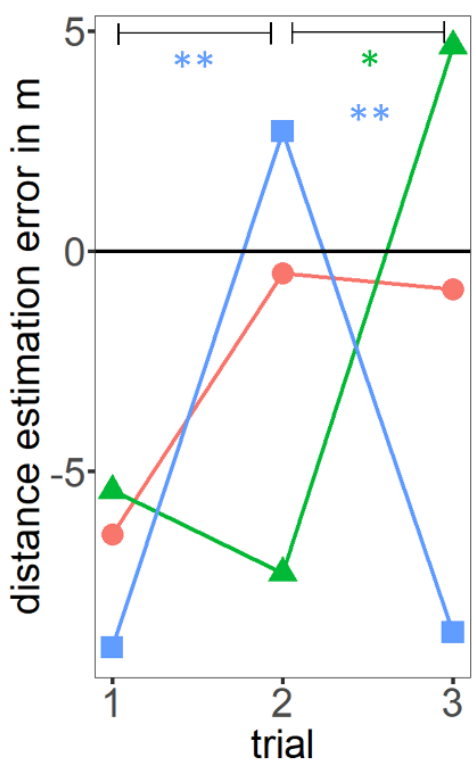

\section{condition}

\section{$5 / 5 / 5 \mathrm{~km} / \mathrm{h}$}

- $5 / 7 / 3 \mathrm{~km} / \mathrm{h}$ $5 / 3 / 7 \mathrm{~km} / \mathrm{h}$
$=-0.38, \mathrm{p}=.7$ ), but the differences were not statistically significant.

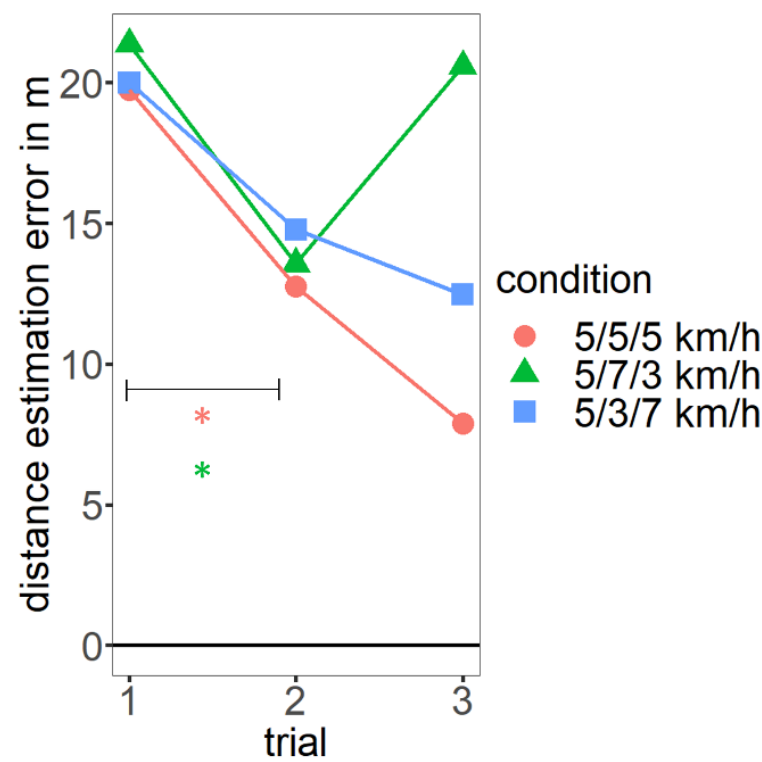

Figure 3. Distance estimation errors per condition and trial. The left graph shows the directional distance estimation errors and the right graph shows the absolute distance estimation errors $\left(* p<.05\right.$, $\left.{ }^{* *} \mathrm{p}<.01\right)$.

\section{Discussion and conclusions}

The results indicate that variations of locomotion speed indeed affected distance estimations. This extends previous single screen-based studies on motion adaptation (Hoffmann et al., 1999; Schrater \& Simoncelli, 1998), as we found first evidence that motion adaptation also occurs during VR locomotion. However, opposed to our expectations and in contradiction to Hietanen et al. (2008), distance estimations were lower after the locomotion speed was increased and higher after the locomotion speed was decreased. This unexpected reverse pattern of distance estimations indicates that subjective travel duration might play a greater role for distance estimations than subjective travel speed.

In agreement with previous studies that report a general trend towards underestimating distances when visually perceived locomotion is not supported by proprioceptive or vestibular feedback (Campos et al., 2012, 2014; Keil, Edler, O'Meara, et al., 2021), distances were generally underestimated in the first trial. Albeit only the absolute error differences were statistically significant, in agreement with the findings of Keil, Edler, O'Meara, et al. (2021), providing distance estimation trainings shifted underestimations closer to an accurate estimation if the locomotion speed was kept constant.

The fact that motion adaptation effects are more pronounced in condition three compared to condition two, especially between trial one and two (see Figure 3), indicates that effects of motion adaptation might have interacted with effects of the provided distance estimation training. Higher distance estimations based on a training, due to a general tendency to underestimate distances without a training, seem to add up with higher estimations if the locomotion speed is reduced, causing overestimations of traveled distances. Shorter distance estimations due to an increased locomotion speed on the other hand seem to be canceled out by the training-based trend for higher estimations. Thus, we can confirm our assumption that distance estimation trainings are less suitable for reducing distance estimation errors if the locomotion speed varies. Still, the similar trend of absolute distance estimation errors in the conditions one $(5 / 5 / 5$ $\mathrm{km} / \mathrm{h})$ and three $(5 / 3 / 7 \mathrm{~km} / \mathrm{h}$, see Figure 3$)$ indicates that training effects might, at least partially, have helped to improve distance estimations.

The findings demonstrate that the ability of VR users to choose an artificial locomotion speed dynamically can affect the perception of distances in VR environments and, consequentially, might affect the formation of an accurate cognitive representation of the perceived space. Therefore, in cases where the acquisition of accurate spatial information is highly important, VR developers should implement fixed locomotion speeds and provide a distance estimation training. In order to deepen our understanding of the identified motion adaptation effects, follow-up studies need to investigate how travel duration affects distance estimations in VR with different locomotion speeds and travel distances. 


\section{References}

Boletsis, C., \& Cedergren, J. E. (2019). Vr locomotion in the new era of virtual reality: An empirical comparison of prevalent techniques. Advances in HumanComputer Interaction, 2019, 1-15. https://doi.org/10.1155/2019/7420781

Campos, J. L., Butler, J. S., \& Bülthoff, H. H. (2012). Multisensory integration in the estimation of walked distances. Experimental Brain Research, 218(4), 551565. https://doi.org/10.1007/s00221-012-3048-1

Campos, J. L., Butler, J. S., \& Bülthoff, H. H. (2014). Contributions of visual and proprioceptive information to travelled distance estimation during changing sensory congruencies. Experimental Brain Research, 232(10), 3277-3289. https://doi.org/10.1007/s00221014-4011-0

Gray, R., \& Regan, D. (2000). Risky driving behavior: A consequence of motion adaptation for visually guided motor action. Journal of Experimental Psychology: Human Perception and Performance, 26(6), 17211732. https://doi.org/10.1037/0096-1523.26.6.1721

Hietanen, M. A., Crowder, N. A., \& Ibbotson, M. R. (2008). Differential changes in human perception of speed due to motion adaptation. Journal of Vision, 8(11), 1-10. https://doi.org/10.1167/8.11.6

Hoffmann, M., Dorn, T. J., \& Bach, M. (1999). Time course of motion adaptation: Motion-onset visual evoked potentials and subjective estimates. Vision Research, 39(3), 437-444. https://doi.org/10.1016/S0042-6989(98)00186-2

Hruby, F., Castellanos, I., \& Ressl, R. (2020). Cartographic scale in immersive virtual environments. KN - Journal of Cartography and Geographic Information, 71(1), 45-51. https://doi.org/10.1007/s42489-020-00055-y

Keil, J., Edler, D., O'Meara, D., Korte, A., \& Dickmann, F. (2021). Effects of virtual reality locomotion techniques on distance estimations. ISPRS International Journal of Geo-Information, 10(3), 1-17. https://doi.org/10.3390/ijgi10030150

Keil, J., Edler, D., Schmitt, T., \& Dickmann, F. (2021). Creating immersive virtual environments based on open geospatial data and game engines. KN - Journal of Cartography and Geographic Information, 71(1), 53-65. https://doi.org/10.1007/s42489-020-00069-6

Langbehn, E., Lubos, P., \& Steinicke, F. (2018). Evaluation of locomotion techniques for room-scale vr: Joystick, teleportation, and redirected walking. In S. Richir (Ed.), Proceedings of the virtual reality international conference - laval virtual on - vric ' 18 (pp. 1-9). ACM Press. https://doi.org/10.1145/3234253.3234291

Schrater, P. R., \& Simoncelli, E. P. (1998). Local velocity representation: Evidence from motion adaptation. Vision Research, 38(24), 3899-3912. https://doi.org/10.1016/S0042-6989(98)00088-1
So, R. H., Lo, W. T., \& Ho, A. T. (2001). Effects of navigation speed on motion sickness caused by an immersive virtual environment. Human Factors, 43(3), $452-461$. https://doi.org/10.1518/001872001775898223 